\title{
Xuebijing injection in the treatment of COVID-19: a retrospective case-control study
}

\author{
Hu Guo ${ }^{1 \#}$, Jiangyuan Zheng ${ }^{2 \#}$, Gu Huang ${ }^{1 \#}$, Yi Xiang ${ }^{3}$, Chunhui Lang ${ }^{4}$, Boqun Li $^{1}$, Daoqiu Huang ${ }^{1}$, \\ Qiuyan Sun ${ }^{1}$, Yaling Luo $^{2}$, Yulian Zhang ${ }^{1}$, Liang Huang ${ }^{5}$, Wei Fang ${ }^{1}$, Yu Zheng ${ }^{6}$, Suxin Wan ${ }^{1 \wedge}$ \\ ${ }^{1}$ Pharmaceutical Department of Chongqing Three Gorges Central Hospital, Chongqing University Three Gorges Hospital, Chongqing, China; \\ ${ }^{2}$ College of Medical Information, Chongqing Medical University, Chongqing, China; ${ }^{3}$ Liver Center, Yu An Branch of Chongqing Three Gorges \\ Central Hospital, Chongqing University Three Gorges Hospital, Chongqing, China; ${ }^{4}$ Research and Foreign Affairs Department of Chongqing \\ Three Gorges Central Hospital, Chongqing University Three Gorges Hospital, Chongqing, China; ${ }^{5}$ Cardiology of Chongqing Three Gorges \\ Central Hospital, Chongqing University Three Gorges Hospital, Chongqing, China; ${ }^{6}$ Pharmacy College, Chengdu University of Traditional \\ Chinese Medicine, Key Laboratory of Standardization of Chinese Herbal Medicine, Ministry of Education, Key Laboratory of Systematic Research, \\ Development and Utilization of Chinese Medicine Resources in Sichuan Province, Key Laboratory Breeding Base of Co-founded by Sichuan \\ Province and Ministry of Science and Technology, Chengdu, China \\ Contributions: (I) Conception and design: H Guo, S Wan, G Huang; (II) Administrative support: C Lang, B Li, D Huang, Q Sun, W Fang; (III) \\ Provision of study materials or patients: All authors; (IV) Collection and assembly of data: H Guo, G Huang, Y Zhang, S Wan; (V) Data analysis and \\ interpretation: J Zheng, Y Luo, S Wan; (VI) Manuscript writing: All authors; (VII) Final approval of manuscript: All authors. \\ "These authors contributed equally to this work as co-first authors. \\ Correspondence to: Suxin Wan. Pharmaceutical Department of Chongqing Three Gorges Central Hospital, Chongqing University Three Gorges \\ Hospital, Chongqing, China. Email: wansuxin@foxmail.com; Yu Zheng. Pharmacy College, Chengdu University of Traditional Chinese Medicine, \\ Key Laboratory of Standardization of Chinese Herbal Medicine, Ministry of Education, Key Laboratory of Systematic Research, Development and \\ Utilization of Chinese Medicine Resources in Sichuan Province, Key Laboratory Breeding Base of Co-founded by Sichuan Province and Ministry of \\ Science and Technology, Chengdu, China. Email: yuzheng1@cdutcm.edu.cn; Wei Fang. Pharmaceutical Department of Chongqing Three Gorges \\ Central Hospital, Chongqing University Three Gorges Hospital, Chongqing, China. Email: 239491815@qq.com.
}

Background: Neither a vaccine nor specific therapeutic drugs against 2019 novel coronavirus have been developed. Some studies have shown that Xuebijing injection (XBJ) can exert an anti-inflammatory effect by inhibiting the production of interleukin-6 (IL-6), tumor necrosis factor alpha (TNF- $\alpha$ ), and other cytokines. This study aimed to investigate the effect of XBJ on coronavirus disease 2019 (COVID-19) and its effects on IL-6 and tumor necrosis alpha TNF- $\alpha$.

Methods: A total of 42 patients, who were diagnosed with COVID-19 and treated with XBJ combined with routine treatment at Chongqing University Three Gorges Hospital between January 20, 2020, and March 11, 2020, were selected as the observation group. A control group comprising 16 patients who received routine treatment was also established, and cases were matched from the observation group on a 1:1 basis according to age, comorbidities, and mild and severe disease. The clinical symptoms, laboratory test indexes, and changes in computed tomography (CT) scans of patients in the two groups were observed at the time of admission and 7 days after treatment, and the time taken for the patients to produce a negative nucleic acid test was also recorded.

Results: There were no significant differences in baseline data between the two groups. After treatment, there were significant improvements in IL-6 levels and body temperature in the observation group as compared with the control group. Particularly in severe patients, the reduction in body temperature in the observation group was greater than that in the control group $(\mathrm{P}<0.05)$. A higher number of patients in the observation group showed improved CT imaging results compared with the control group, and the time taken to produce a negative nucleic acid test was shorter in the observation group than in the control group;

$\wedge$ ORCID: 0000-0003-1742-2018. 
however, the differences were not statistically significant $(\mathrm{P}>0.05)$. Furthermore, there were no significant differences in TNF- $\alpha$ and IL-10 between the two groups.

Conclusions: The results of this study suggest that routine treatment combined with XBF can better improve the clinical outcomes of COVID-19 patients.

Keywords: Xuebijing injection; coronavirus disease 2019 (COVID-19); effect; interleukin 6 (IL-6)

Submitted Jun 09, 2020. Accepted for publication Aug 27, 2020.

doi: 10.21037/apm-20-1478

View this article at: http://dx.doi.org/10.21037/apm-20-1478

\section{Introduction}

Coronavirus disease 2019 (COVID-19) is caused by a novel coronavirus, severe acute respiratory syndrome coronavirus 2 (SARS-CoV2), a new zoonotic agent that was initially known as 2019 novel coronavirus (2019-nCoV) (1-3). By 28 July, 2020, the World Health Organization (WHO) had reported more than 16,185,037 confirmed cases and over 644,972 deaths. The threat posed by the disease to global public health led the WHO to declare COVID-19 as a public health emergency of international concern (PHEIC). Currently, there are many studies on the treatment of COVID-19. However, neither a vaccine nor specific therapeutic drugs against this communicable disease have been developed (4). Xuebijing injection (XBJ) is one of the therapeutic drugs recommended in the practice guidelines published by the Chinese Health Commission. Few clinical studies on the treatment of COVID-19 by XBJ have been found. This study conducted a preliminary clinical study on the treatment of COVID-19 with XBJ. And a better understanding of SARS-CoV2 is fundamental for the discovery of effective vaccines and drugs $(5,6)$.

$\mathrm{XBJ}$ is an important form of therapy in traditional Chinese medicine (TCM). XBJ consists of Carthamus tinctorius, Radix Paeoniae Rubra, Ligusticum wallichii, Salvia miltiorrhiza, and Angelica sinensis (7). Containing multiple active ingredients, such as safflower yellow A, ligustrazine, danshensu, ferulic acid, paeoniflorin, and protocatechualdehyde, XBJ dissipates blood stasis by activating blood circulation, thus eliminating pathogenic heat from the blood and degrading toxins. By improving microcirculation, these ingredients perform a marked function in coagulation dysfunction, inhibiting platelet adhesion and aggregation. Clinically, XBJ is mainly used for the treatment of sepsis, infection-induced systemic inflammatory response syndrome (SIRS), and multiple organ dysfunction syndrome (MODS) $(8,9)$. Approved as a class II new drug in China since 2004, XBJ was successively included in the "Diagnosis and treatment plan for New Coronavirus Pneumonia (Trial version 4, 5, 6 and 7)" and "Diagnosis and treatment plan for New Coronavirus Pneumonia in severe and critical cases (Trial version 2) issued by the National Health Commission of the People's Republic of China and the National Administration of Traditional Chinese Medicine $(10,11)$. The injection has been included in the treatment guidelines for a number of viral infectious diseases in China, and recommended as a treatment for sepsis and septic shock (12). In Severe Community Acquired Pneumonia, XBJ injection has been shown to significantly improve the primary endpoint statistics of the pneumonia severity index and secondary clinical outcomes such as mortality, mechanical ventilation time, and duration $(13,14)$. Therefore, this study aimed to observe the effect of XBJ injection in the treatment of COVID-19.

We present the following article in accordance with the STROBE reporting checklist (available at http://dx.doi. org/10.21037/apm-20-1478).

\section{Methods}

\section{Study design}

A retrospective case analysis was performed. The study was approved by the Ethics Commission of the Chongqing University Three Gorges Hospital (Chongqing Three Gorges Central Hospital), and was in accordance with the ethical principles of the Declaration of Helsinki (as revised in 2013). Because of the retrospective nature of the research, the requirement for informed consent was waived. The study analyzed 42 cases diagnosed with COVID-19 and treated with $\mathrm{XBJ}$ injection as well as 16 cases (the 
control group) treated with routine treatment, in the Three Gorges Hospital of Chongqing University between January 20, 2020, and March 11, 2020. The underlying disease is indeed an important factor affecting the prognosis of many diseases, as is COVID-19. Currently, many studies have shown that basic diseases such as age, cardiovascular and diabetes have a greater impact on the prognosis of COVID-19 $(15,16)$. Therefore, in order to minimize the impact of basic diseases on the study results, the cases were matched 1:1 according to age, basic diseases and severe cases in this study.

\section{Inclusion and exclusion criteria}

The inclusion criteria for cases were as follows: (I) aged $>18$ years old; (II) meeting the diagnostic criteria of "Diagnosis and treatment plan for the New Coronavirus Pneumonia (Trial Seventh Edition)"; and (III) hospitalized for $\geq 7$ days. The observation group must have received continuous treatment with XBJ injection for more than 7 days.

The exclusion criteria were as follows: (I) observation group: treatment with XBJ injection lasted less than 7 days; (II) control group: could not be matched to patients in the observation group according to age, comorbidity, or disease severity; or (III) a lack of significant outcome indicators.

\section{Case collection}

The research team collected the clinical data of COVID-19 patients from January 20, 2020, to March 11, 2020, using the hospital's electronic health information system (HIS). These data included the patients' epidemiological history, medical history, symptoms and signs, laboratory test results, chest CT findings, and treatment measures.

\section{Classification of mild and severe cases}

According to the "Diagnosis and treatment plan for the New Coronavirus Pneumonia (Trial Seventh Edition)" issued by the National Health Commission of People's Republic of China and the National Administration of Traditional Chinese Medicine, the patients were divided into mild type (mild and common) and severe type (severe and critical). The mild patients did not have severe symptoms, and no pneumonia was found in imaging, while the common patients had fever as well as respiratory and other symptoms, and the manifestation of pneumonia could be seen in imaging. Patients meeting any of the following criteria were considered as severe: (I) Shortness of breath, $\mathrm{RR} \geq 30$ times/min; (II) oxygen saturation $\leq 93 \%$ in the resting state; or (III) arterial partial pressure of oxygen $\left(\mathrm{PaO}_{2}\right)$ /oxygen concentration $\left(\mathrm{FiO}_{2}\right) \leq 300 \mathrm{mmHg}$. When pulmonary imaging showed that the lesions had progressed by more than 50\% within 24-48 hours, the patients were treated according to the Diagnosis and treatment plan for the New Coronavirus Pneumonia (Trial Seventh Edition). Patients were considered to be critical if they met any of the following criteria: (I) respiratory failure requiring mechanical ventilation; (II) shock; or (III) other organ failure requiring ICU monitoring.

\section{Interventions}

All patients were given routine treatment according to the "Diagnosis and treatment plan for the New Coronavirus Pneumonia", including electrolyte balance, blood glucose and blood pressure management, nutritional support, oxygen therapy, and antiviral treatment (Interferon- $\alpha$, Lopinavir/litonavir, Chloroquine, Ribavirin, Abidor). The observation group was given XBJ injections (Tianjin HongRi Pharmaceutical Co., Ltd.; gyzz20040033) on the basis of routine treatment $(100 \mathrm{~mL}+0.9 \% \mathrm{~ns} 100 \mathrm{~mL}$, twice a day; each infusion was completed within 30 minutes).

\section{Evaluation index}

The changes in outcome indexes were compared before and 7 days after treatment. The main outcome measures were: length of hospital stay; duration of mechanical ventilation; the time taken for the patient to produce a negative nucleic acid test (if the results were negative twice in a row, the first test was included); relief of clinical symptoms; leukocyte, neutrophil, and platelet counts; absolute lymphocyte value; C-reactive protein (CRP); interleukin-6 (IL-6); interleukin-10 (IL-10); tumor necrosis factor- $\alpha$ (TNF- $\alpha$ ); fibrinogen; thromboplastin time (TT); prothrombin time (PT); D-dimer; lactate; creatinine; and chest CT examination.

\section{Statistical analysis}

The categorical variables were described as percentages, and the continuous variables were described as the values of the mean, median, and interquartile range (IQR). The outcome indicators in the observation and control groups before 
Table 1 General information of the 42 patients

\begin{tabular}{|c|c|}
\hline Items & All patients \\
\hline Age [median (IQR), y] & $52.75[64-78]$ \\
\hline \multicolumn{2}{|l|}{ Sex, n (\%) } \\
\hline Men & $21(50.0)$ \\
\hline Women & $21(50.0)$ \\
\hline \multicolumn{2}{|l|}{ Any comorbidity, n (\%) } \\
\hline Cardiovascular disease & $8(19.0)$ \\
\hline Diabetes & $6(14.3)$ \\
\hline Chronic kidney disease & $1(2.4)$ \\
\hline \multicolumn{2}{|l|}{ CT results after treatment $(n=40), n(\%)$} \\
\hline Improvement & $24(60.0)$ \\
\hline Aggravation & $11(27.5)$ \\
\hline No significant changes & $5(12.5)$ \\
\hline $\begin{array}{l}\text { Time of nucleic acid conversion to negative } \\
{[n \text {, median (IQR), d] }}\end{array}$ & $38,10(7-14.5)$ \\
\hline $\begin{array}{l}\text { Mechanical ventilation time [n, median (IQR), } \\
\text { d] }\end{array}$ & $14,6(2.5-9)$ \\
\hline Length of hospital stay [n, median (IQR), d] & $42,16.5[12-22]$ \\
\hline
\end{tabular}

and after treatment were compared by adopting the selfcontrast and inter-contrast methods, and the comparison was made by further grouping the patients according to mild and severe disease. Continuous variables with normal distribution were compared using independent group $t$-tests; otherwise, the Mann-Whitney $U$ test or Wilcoxon test was used. The $\chi^{2}$ test was used to compare the proportions of categorical variables. For unadjusted comparisons, a twosided $\alpha$ of $<0.05$ was considered to be statistically significant. For multiple comparisons, the analyses were not adjusted, and given the potential for type I error, the findings should be interpreted as exploratory and descriptive. SPSS 22.0 software (IBM, Armonk, New York, USA) was used for all statistical analyses.

\section{Results}

\section{Patients}

According to the inclusion and exclusion criteria, 42 patients who were treated with XBJ injection were included as the observation group in this study. The differences of the outcome indicators before and after treatment were analyzed. Meanwhile, 1:1 matched casecontrol studies of 42 patients in the observation group were performed according to age, complications, and disease severity, and 16 of them were matched to the appropriate control. Therefore, a total of 32 patients were included in the matched-pair analysis (16 in the observation group and 16 in the control group).

\section{Baseline information and self-contrast analysis of outcome indexes before and after XBF treatment of 42 patients}

Among the 42 patients treated with XBJ injection, 8 cases were mild, 34 cases were severe. There were 21 males and 21 females, ranging in age from 25 to 87 years old, with a median and quartile of 52.75 [64-78] years old. Among them, 15 patients had pre-existing conditions including 8 cases of cardiovascular disease, 6 cases of diabetes, and 1 case of kidney disease. Mechanical ventilation was used in 14 cases, with a median mechanical ventilation of $6(2.5-9)$ days. CT imaging of 24 cases (60\%) showed improvement after 7 days of XBJ injection combined with conventional treatment. The 42 patients had a median length of hospital stay of 16.5 [12-22] days, and the median time taken to produce a negative nucleic acid test was $10(7-14.5)$ days, as shown in Table 1.

Paired tests of outcome indicators of all patients before and after treatment revealed significant differences in white blood cell count, neutrophil count, platelet count, CRP, IL-6, TNF- $\alpha$, fibrinogen, and D-dimer $(\mathrm{P}<0.05)$. Among them, the white blood cell count, neutrophil count, platelet count, and fibrinogen were all increased after treatment, while CRP, IL-6, and d-dimer were all decreased (Table 2).

\section{General information of 32 paired patients}

The general data of the 32 paired patients (16 from the observation group and 16 from the control group) are shown in Table 3. Among the 16 patients from the observation group, 8 were mild and 8 were severe. There were 9 males $(56.25 \%)$, and the median and quartile values of age were 52 [46-57] years old. Of these patients, 7 had pre-existing conditions (5 cases had cardiovascular diseases and 2 cases had diabetes). Among the 16 patients in the control group, 8 were mild and 8 were severe. There were 11 males $(68.75 \%)$, and the median and quartile values of age were 54 [44-59] years old. Of these patients, 7 had preexisting conditions (5 cases had cardiovascular diseases and 2 cases had diabetes). 
Table 2 Analysis of outcome indicators before and after treatment of the 42 patients

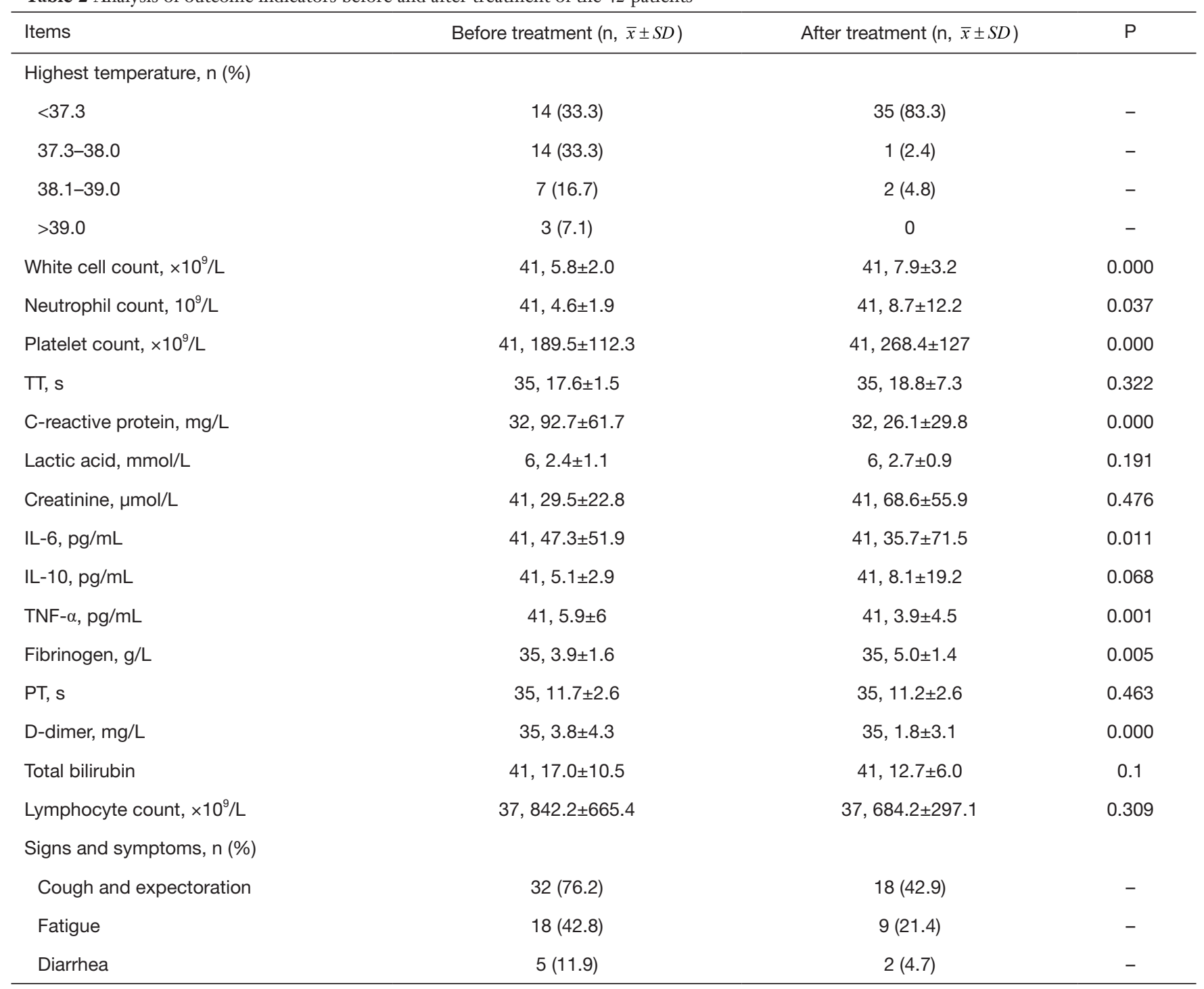

Table 3 General information of the 32 matched patients

\begin{tabular}{|c|c|c|c|c|c|c|}
\hline Items & \multicolumn{3}{|c|}{ Observation group } & \multicolumn{3}{|c|}{ Control group } \\
\hline Age [median (IQR), y] & $52[46-57]$ & 54 [40-67] & $50[46-56]$ & 54 [44-59] & 54 [41-62] & 53 [44-59] \\
\hline \multicolumn{7}{|l|}{ Sex, n (\%) } \\
\hline Men & $9(56.25)$ & $4(25.0)$ & $5(31.25)$ & $11(68.75)$ & $5(31.25)$ & $6(37.5)$ \\
\hline \multicolumn{7}{|l|}{ Any comorbidity, n (\%) } \\
\hline Cardiovascular disease & $5(31.25)$ & $4(25.0)$ & $1(6.25)$ & $5(31.25)$ & $4(25.0)$ & $1(6.25)$ \\
\hline Diabetes & $2(12.5)$ & 0 & $2(12.5)$ & $2(12.5)$ & 0 & $2(12.5)$ \\
\hline
\end{tabular}


Table 4 Analysis of outcome indicators before and after treatment in the observation group

\begin{tabular}{|c|c|c|c|c|c|c|c|c|c|}
\hline Index & \multicolumn{3}{|c|}{ Total number $(n=16)$} & \multicolumn{3}{|c|}{ Mild cases $(n=8)$} & \multicolumn{3}{|c|}{ Severe cases $(n=8)$} \\
\hline $\begin{array}{l}\text { White cell } \\
\text { count, } \times 10^{9} / \mathrm{L}\end{array}$ & $15,5.6 \pm 2.5$ & $15,7.6 \pm 4.1$ & 0.023 & $8,5.5 \pm 2.1$ & $8,6.8 \pm 2.8$ & 0.121 & $7,5.7 \pm 3.0$ & $7,8.6 \pm 5.3$ & 0.098 \\
\hline $\begin{array}{l}\text { Platelet count, } \\
\times 10^{9} / \mathrm{L}\end{array}$ & $15,198.4 \pm 79.9$ & $15,282.9 \pm 98.2$ & 0.013 & $8,206.3 \pm 94.4$ & $8,282.0 \pm 97.9$ & 0.192 & $7,189.4 \pm 65.9$ & $7,284.0 \pm 106.3$ & 0.014 \\
\hline$\pi, s$ & $13,17.2 \pm 1.3$ & $13,17.1 \pm 1.4$ & 0.824 & $7,17.6 \pm 1.2$ & $7,18.0 \pm 0.8$ & 0.594 & $6,16.7 \pm 1.2$ & $6,16.1 \pm 1.2$ & 0.375 \\
\hline $\begin{array}{l}\text { Creatinine, } \\
\mu \mathrm{mol} / \mathrm{L}\end{array}$ & $15,68.4 \pm 28.5$ & $15,61.9 \pm 25$ & 0.128 & $8,69.6 \pm 25.2$ & $8,59.1 \pm 10.7$ & 0.166 & $7,67.0 \pm 34.0$ & $7,65.0 \pm 36.1$ & 0.344 \\
\hline IL-6, pg/mL & $15,37.5 \pm 48.8$ & $15,10.2 \pm 9.5$ & 0.002 & $8,51.6 \pm 64.2$ & $8,10.9 \pm 9.9$ & 0.017 & $7,21.3 \pm 13.4$ & $7,9.3 \pm 9.8$ & 0.043 \\
\hline $\mathrm{IL}-10, \mathrm{pg} / \mathrm{mL}$ & $15,4.9 \pm 2.3$ & $15,3.2 \pm 0.6$ & 0.003 & $8,5.3 \pm 2.5$ & $8,3.3 \pm 0.6$ & 0.037 & $7,4.5 \pm 2.2$ & $7,3.1 \pm 0.6$ & 0.028 \\
\hline TNF- $\alpha, p g / m L$ & $15,5.7 \pm 4.3$ & $15,4.3 \pm 2.6$ & 0.125 & $8,5.6 \pm 4.9$ & $8,3.7 \pm 1.4$ & 0.161 & $7,5.8 \pm 3.9$ & $7,5.1 \pm 3.5$ & 0.309 \\
\hline $\begin{array}{l}\text { Fibrinogen, } \\
\text { g/L }\end{array}$ & $13,5.6 \pm 1.3$ & $13,4.3 \pm 1.5$ & 0.07 & $7,5.3 \pm 1.3$ & $7,3.9 \pm 1.5$ & 0.168 & $6,5.9 \pm 1.2$ & $6,4.8 \pm 1.4$ & 0.5 \\
\hline PT, s & $13,11.1 \pm 0.8$ & $13,11.1 \pm 0.8$ & 0.6 & $7,11.4 \pm 0.8$ & $7,11.3 \pm 0.6$ & 0.735 & $6,10.8 \pm 0.7$ & $6,10.9 \pm 1.0$ & 0.834 \\
\hline
\end{tabular}

Results of self-control analysis of outcome indicators in the observation group and the control group before and after treatment

The results of analysis of outcome indicators in 16 patients in the observation group before and after treatment are shown in Table 4. The results of simultaneous analysis of outcome indicators in mild and severe patients showed that there were significant differences in white blood cell count, neutrophil count, platelet count, CRP, IL-6, and IL-10 $(\mathrm{P}<0.05)$. Among these indicators, leukocyte count, neutrophil count, and platelet count were increased, while CRP, IL-6, and IL-10 were decreased after treatment. The results of simultaneous analysis of outcome indicators in mild and severe patients showed that the levels of IL-6 and IL-10 were lower after treatment than before treatment in mild patients $(\mathrm{P}<0.05)$. In severe patients, the platelet count was increased after treatment $(\mathrm{P}<0.05)$, and the levels of both IL- 6 and IL-10 were decreased compared with those before treatment $(\mathrm{P}<0.05)$, as shown in Table 4 .

The results of analysis of outcome indicators in 16 patients in the control group before and after treatment are shown in Table 5. The results showed that PLT was increased after treatment compared with before treatment $(\mathrm{P}<0.05)$. The results of separate analysis of outcome indicators in mild and severe patients showed that there were no significant differences in any of the indexes of the mild patients; however, for the severe patients, the PLT 
Table 5 Analysis of outcome indicators before and after treatment in the control group

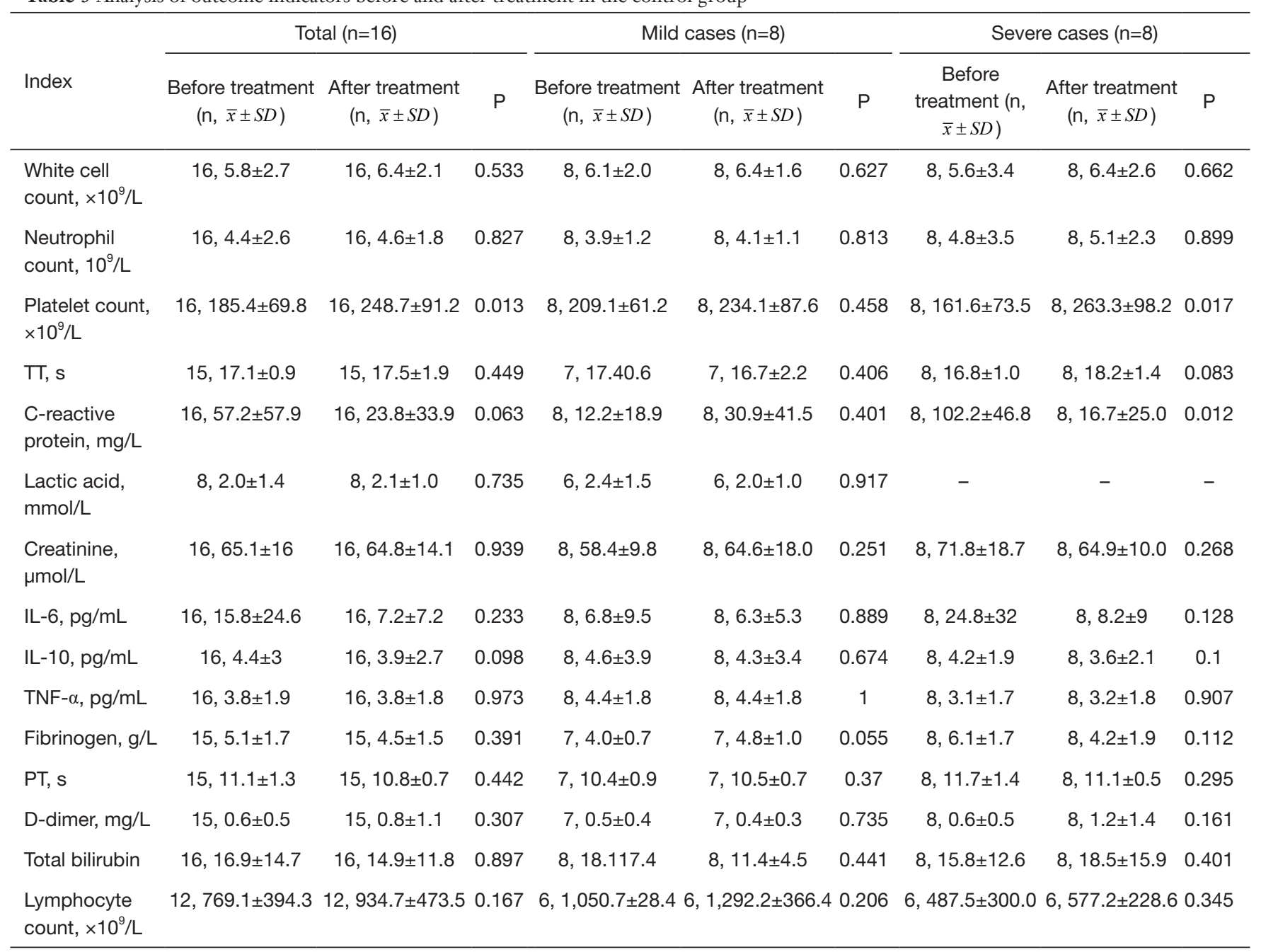

after treatment was higher than that before treatment $(\mathrm{P}<0.05)$, and the CRP was lower than that before treatment $(\mathrm{P}<0.05)$, as shown in Table 5 .

\section{Analysis of outcome indicators between the observation group and the control group before and after treatment}

Before treatment, 15 of the 32 paired patients had a fever, and the number of severe patients with fever in the observation group was lower than that in the control group $(\mathrm{P}<0.05)$. Following treatment, 11 of the 32 patients still had a fever, and the number in the control group was higher than that in the observation group $(\mathrm{P}<0.05)$. Meanwhile, the number of severe patients in the control group was also higher than that in the observation group $(\mathrm{P}<0.05)$, as shown in Table 6. There were no significant differences in cough, sputum, fatigue, or diarrhea before and after treatment between the two groups. After treatment, there were no significant differences between the two groups in CT results, total length of hospital stay, or the time taken to produce a negative nucleic acid test. However, whether the two groups were compared directly or analyzed separately, the CT results were better in the observation group than in the control group, and the time taken to produce a negative nucleic acid test was shorter in the observation group.

Table 7 shows the results of analysis of the 32 patients. Before treatment, D-dimer was higher in the observation group than in the control group, which was the only significant difference between the groups $(\mathrm{P}<0.05)$. After treatment, only the level of IL-6 was significantly different between the two groups and was lower in the observation group than in the control group $(\mathrm{P}<0.05)$. 
Table 6 Analysis of outcome indicators before and after treatment in observation group and control group

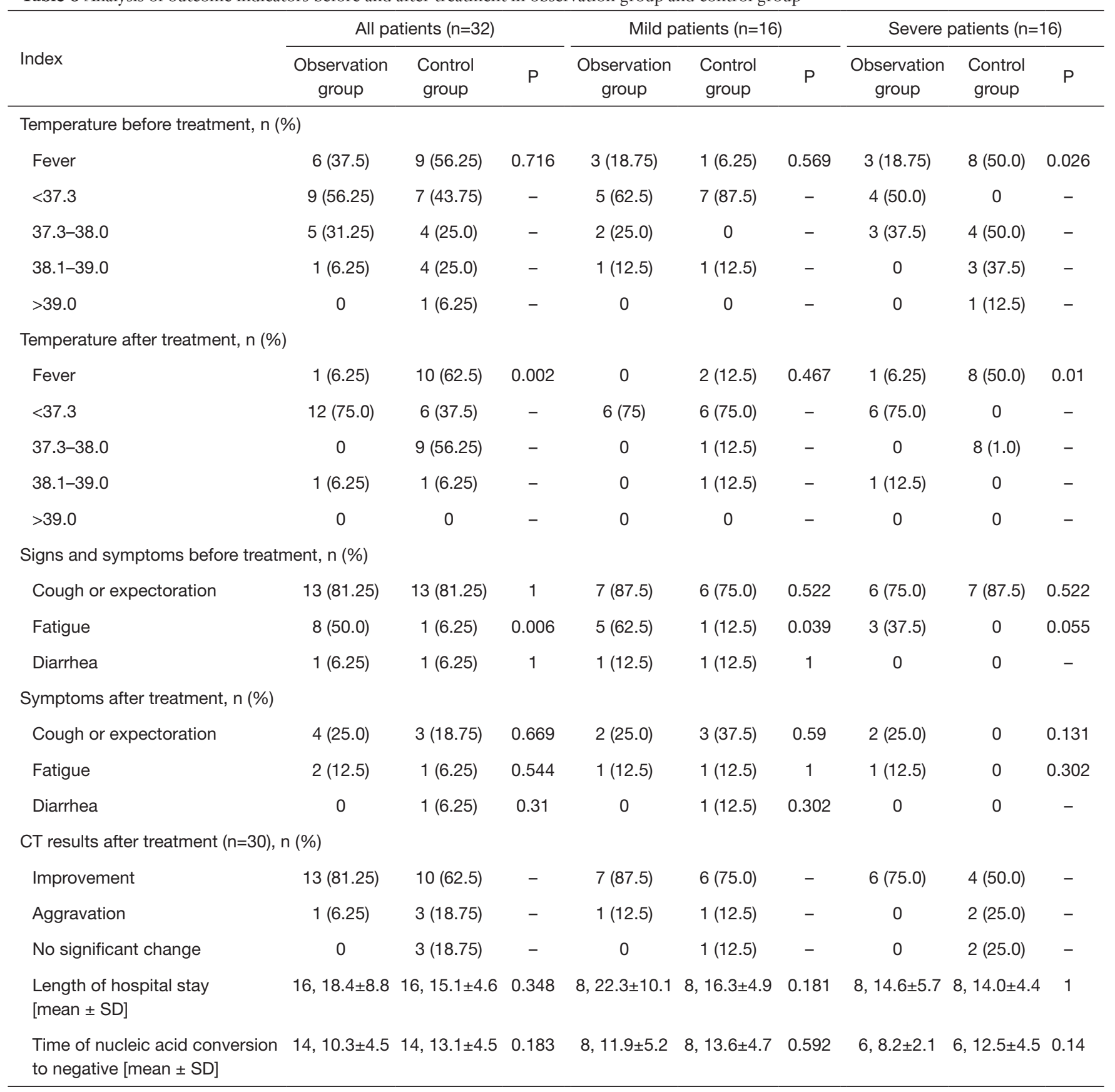

The patients in the observation group and the control group were then grouped according to disease severity, and the outcome indicators before and after treatment were calculated for each group, as shown in Table 8 . In mild patients, fibrinogen and D dimer were higher in the observation group than in the control group before treatment $(\mathrm{P}<0.05)$. After treatment, the lymphocyte counts in both groups were increased and the level of IL-6 was decreased. After treatment, the lymphocyte count of mild patients in the observation group was lower than that in the control group $(\mathrm{P}<0.05)$. Meanwhile, in both mild and severe patients, the level of IL-6 in the observation group was lower than that in the control group $(\mathrm{P}<0.05)$. In severe patients, there were no significant differences in any of the 
Table 7 Analysis of outcome indicators before and after treatment in observation group and control group

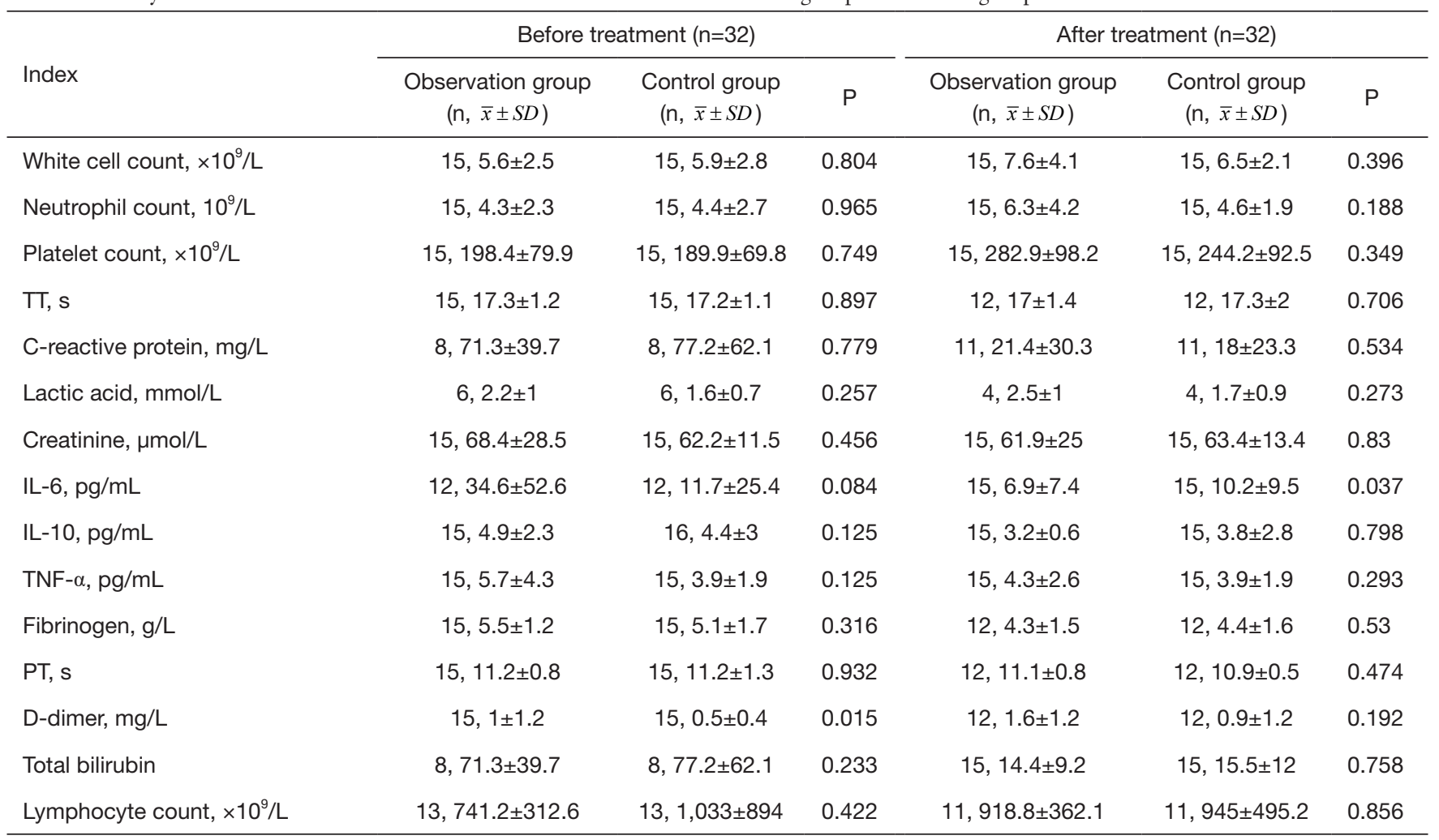

indicators between the observation group and the control group before treatment, while after treatment, the level of IL-6 in the observation group was lower than that in the control group $(\mathrm{P}<0.05)$.

\section{Discussion}

COVID-19 is a respiratory tract infection caused by a novel coronavirus that is believed to have originated as a zoonotic virus that has mutated or otherwise adapted to allow human pathogenicity (17). Currently, several existing antiviral agents are being used in clinical trials and compassionate use protocols based on in vitro activity (against this or related viruses) and on limited clinical experience. Lopinavir-ritonavir is approved by the United States Food and Drug Administration (FDA) for the treatment of human immunodeficiency virus (HIV). It has been used to treat other coronavirus infections; it was used empirically for MERS (18) and is being studied in the treatment of SARS (19). A trial involving 199 patients with COVID-19 comparing lopinavir-ritonavir with standard care did not find a significant difference in time to improvement or in mortality at 28 days, nor were there differences in duration of viral RNA in oropharyngeal specimens (20). Chloroquine and its derivatives have reportedly achieved favorable results after use in China and South Korea, although details are lacking, and further trials are under way (21). Azithromycin combined with hydroxychloroquine has been used in some protocols; however, the risk of cardiac arrhythmias must be considered. At present, no specific antiviral agent has been approved for the treatment of COVID-19.

XBJ was developed by Professor Jinda Wang under the guidance of the combined treatment theory of "bacteria, toxin and inflammation" after three decades of research (11). It possesses anti-inflammatory, coagulation regulation, vascular endothelial protection, immune regulation, and other pharmacological properties, and exerts its antiinflammatory effects mainly through inhibiting the production of IL-6, TNF- $\alpha$, and other cytokine (22-26). IL-6 is a multifunctional cytokine, which is produced by activated $\mathrm{T}$ cells and mononuclear cells. It has the functions of regulating immune response, acute reaction, and hematopoiesis, and plays an important role in the body's immune response to infection. TNF- $\alpha$, one of the most important transmitters involved in the inflammatory response, is produced by various mononuclear macrophages. 


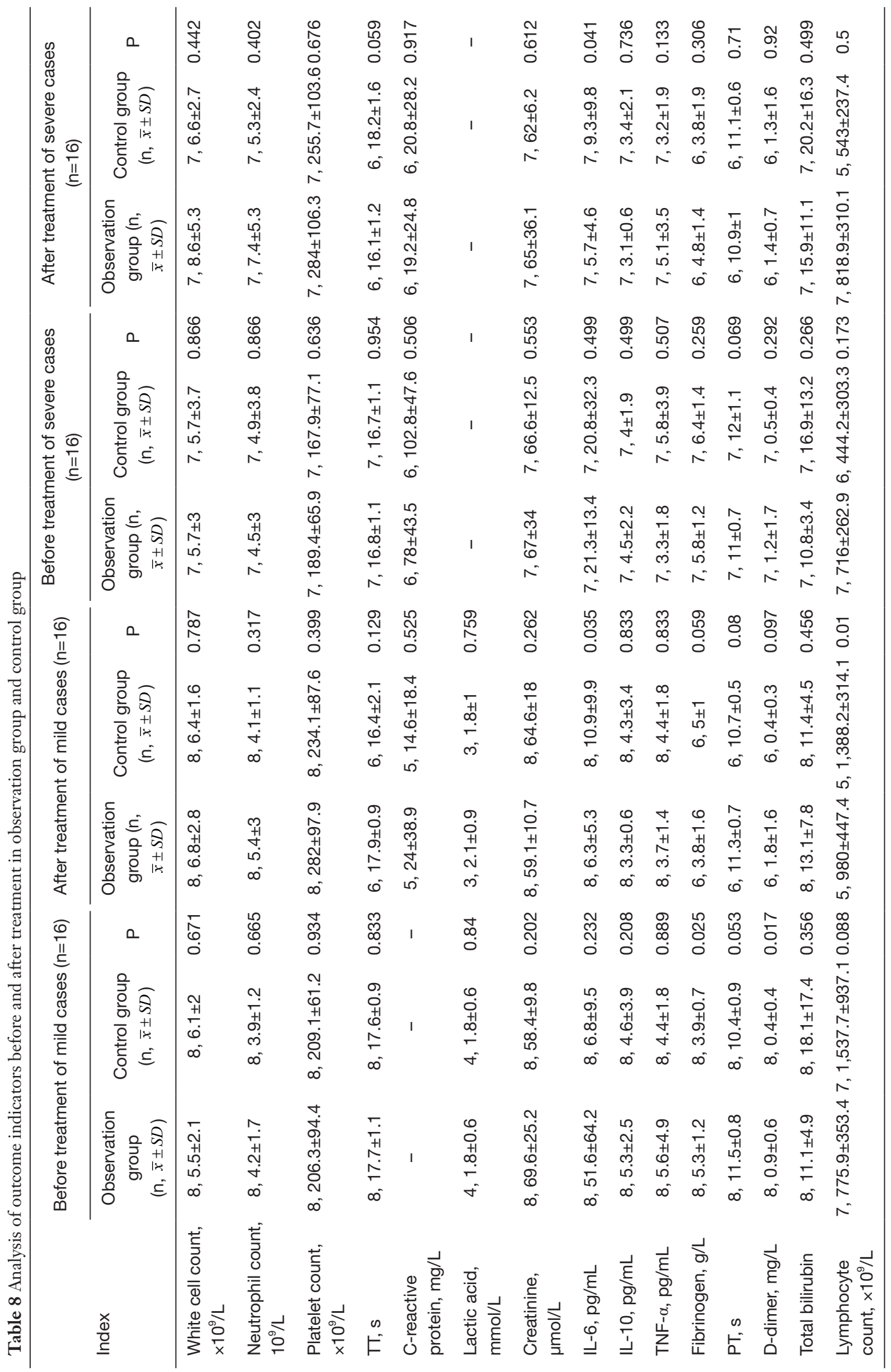


In addition to causing lung damage in a variety of ways, necrosis factor induces and modulates other inflammatory cytokines in the inflammatory response. A high level of TNF- $\alpha$ suggests a more severe illness and worse prognosis. This study found the improvement of interleukin-6 in the observation group was significantly better than that in the control group. It is speculated that the mechanism of XBJ on improving COVID-19 is to inhibit interleukin-6.

Wan et al. confirmed that the level of IL-6 was generally increased in patients with COVID-19, and the level of IL-6 in severe patients was significantly higher than that in mild patients (27). IL-6 and GM-CSF released by T lymphocytes and monocytes may be the key link of the cytokine storm induced by COVID-19 $(28,29)$. Tanaka et al.'s study showed that the reduction of IL-6 indicated a good outcome for the control of cytokine production in sepsis (30).

In this study, after 7 days of combination therapy, the 42 patients in the observation group and 16 patients in the control group showed improvement in all indicators, such as body temperature, cough and sputum symptoms, number of patients with CT improvement, IL-6, CRP, and the improvements in the above indicators in the observation group were better than those in the control group. The results showed that the conventional treatment (oxygen inhalation, antiviral treatment, electrolyte balance, blood glucose and blood pressure management, nutritional support, and immune regulation) achieved a good effect; however, the conventional treatment combined with XBJ had a more obvious effect on improving the conditions of patients with the disease.

Wan and Guan, et al. $(27,29)$ found that age, complications, and disease severity were all important factors affecting the prognosis of COVID-19 patients. Therefore, this study conducted case-control analysis according to these factors. Before treatment, there were no significant differences in the indicators between the two groups, except that the d-dimer of the observation group was higher than that of the control group. This indicated that the baseline data of the two groups were comparable. Seven days after treatment, IL-6 and body temperature were significantly improved in the observation group compared with in the control group. Among severe patients, the improvement in body temperature in the observation group was also better than that in the control group $(\mathrm{P}<0.05)$. At the same time, a higher number of patients in the observation group showed improved CT imaging results compared with the control group, and the time taken for patients in the observation group to produce a negative nucleic acid test was shorter than that in the control group; however, the differences were not statistically significant $(\mathrm{P}>0.05)$. Furthermore, there was no significant difference in TNF- $\alpha$ and IL-10 between the two groups. Another real-world study of 31,913 patients on the centralized monitoring of the clinical safety of XBJ showed that the incidence of adverse reactions (ADR) with this drug was $0.3 \%$, and the adverse reactions observed were mainly those mentioned in the instruction manual. After drug withdrawal and symptomatic treatment, no serious adverse reactions were reported (31).

The results of this study indicated that routine treatment combined with XBJ can better improve the clinical outcomes of patients with COVID-19. Therefore, it can be used as one of the treatment regimens of the disease. However, because the small number of cases admitted to our hospital and the drug was not used in our hospital until after it was included in the Diagnosis and treatment plan for New Coronavirus Pneumonia (Trial version 4) guidelines, the sample size included in this study was small. This is the shortcoming of this research. Therefore, a certain bias existed in the research.

Therefore, in the future, we should collaborate with foreign medical institutions to carry out randomized controlled, multicenter, and large-sample studies on the treatment of COVID-19 with XBJ, so as to further confirm the efficacy and safety of this drug. Tozumab (IL-6 receptor blocker) can effectively reverse the cytokine storm syndrome in the treatment of acute lymphoblastic leukemia (32). So far, this drug has been preliminarily applied in clinical trials for COVID-19 treatment and has achieved encouraging results, and further multi-center clinical trials are under way (33).

COVID-19 has been well controlled in China, but the ongoing global pandemic still calls for a united effort in fighting the disease. How to assess the potential risk of recurrence and reinfection? We think it can be evaluated from the following aspects: (I) $2019 \mathrm{nCoV}$ is a new virus, many of its biological characteristics are unknown, and the recurrence and prolongation of the disease may be its own characteristics. (II) When the patient is discharged from the hospital, the pathogenic microorganisms may not be completely eliminated. The carrier's detoxification amount may not reach the lower limit of existing testing methods. If the immunity is reduced again in the later period, it will cause recurrence or re-infection (34). (III) Previous literature studies have shown that patients with 
underlying diseases such as diabetes and hypertension are more likely to be infected and re-infected with 2019 $\mathrm{nCoV}$, and affect the prognosis. (IV) Because throat swabs are sampled for nucleic acid testing, false negatives may occur. The diagnostic criteria for discharge need to be further explored $(15,16)$. (V) After clinical cure, how strong is the protective effect of antibodies produced by the body? How long is the protection exsist? Currently unknown. Studies have shown that asymptomatic individuals infected with the new coronavirus may only last for two to three months, and those with symptoms may last for a shorter time.

\section{Acknowledgments}

We acknowledge all of the health-care workers who were involved in the diagnosis and treatment of patients in our hospital. We thank our hospital for sharing data, and we thank Prof. Wei Fang, Prof. Boqun Li, and Prof. Qiuyan Sun for administrative support.

Funding: This work is supported by the National Social Science Foundation (No. 15BGL191).

\section{Footnote}

Reporting Checklist: The authors have completed the STROBE reporting checklist. Available at http://dx.doi. org/10.21037/apm-20-1478

Data Sharing Statement: Available at http://dx.doi. org/10.21037/apm-20-1478

Conflicts of Interest: All authors have completed the ICMJE uniform disclosure form (available at http://dx.doi. org/10.21037/apm-20-1478). The authors have no conflicts of interest to declare.

Ethical Statement: The authors are accountable for all aspects of the work in ensuring that questions related to the accuracy or integrity of any part of the work are appropriately investigated and resolved. The study was approved by the Ethics Commission of the Chongqing University Three Gorges Hospital (Chongqing Three Gorges Central Hospital) (No. 2020-22), and was in accordance with the ethical principles of the Declaration of Helsinki (as revised in 2013). Because of the retrospective nature of the research, the requirement for informed consent was waived.
Open Access Statement: This is an Open Access article distributed in accordance with the Creative Commons Attribution-NonCommercial-NoDerivs 4.0 International License (CC BY-NC-ND 4.0), which permits the noncommercial replication and distribution of the article with the strict proviso that no changes or edits are made and the original work is properly cited (including links to both the formal publication through the relevant DOI and the license). See: https://creativecommons.org/licenses/by-nc-nd/4.0/.

\section{References}

1. Xu X, Chen P, Wang J, et al. Evolution of the novel coronavirus from the ongoing Wuhan outbreak and modeling of its spike protein for risk of human transmission. Sci China Life Sci 2020;63:457-60.

2. Wong JEL, Leo YS, Tan CC. COVID-19 in SingaporeCurrent Experience: Critical Global Issues That Require Attention and Action. JAMA 2020;323:1243-4.

3. Li Q, Guan X, Wu P, et al. Early Transmission Dynamics in Wuhan, China, of Novel Coronavirus-Infected Pneumonia. N Engl J Med 2020;382:1199-207.

4. Huang Y, Cai C, Zang J, et al. Treatment strategies of hospitalized patients with coronavirus disease-19. Aging (Albany NY) 2020;12:11224-37.

5. Phua J, Weng L, Ling L, et al. Intensive care management of coronavirus disease 2019 (COVID-19): challenges and recommendations. Lancet Respir Med 2020;8:506-17.

6. Lurie N, Saville M, Hatchett R, et al. Developing Covid-19 Vaccines at Pandemic Speed. N Engl J Med 2020;382:1969-73.

7. Shi H, Hong Y, Qian J, et al. Xuebijing in the treatment of patients with sepsis. Am J Emerg Med 2017;35:285-91.

8. Wang YP, Guo Y, Wen PS, et al. Three Ingredients of Safflower Alleviate Acute Lung Injury and Inhibit NET Release Induced by Lipopolysaccharide. Mediators Inflamm 2020;2020:2720369.

9. Li S, Wang H, Sun Q, et al. Therapeutic Effect of Xuebijing, a Traditional Chinese Medicine Injection, on Rheumatoid Arthritis. Evid Based Complement Alternat Med 2020;2020:2710782.

10. General Office of the National Health and Family Planning Commission. Diagnosis and treatment of pneumonia with a new coronavirus infection (trial version 7). Chinese Journal of Integrated Traditional and Western Medicine 2020:1-3.

11. General Office of the National Health and Family 
Planning Commission. Diagnosis and treatment of pneumonia with a new coronavirus infection. Diagnosis and treatment plan for the New Coronavirus Pneumonia in severe and critical cases (Trial version 2). Chinese Journal of Integrated Traditional and Western Medicine 2020:1-3

12. Li C, Zhang X, Liu S, et al. Current Evidence and Research Prospects of Xuebijing Injection in Treating Novel Coronavirus-infected Pneumonia (COVID-19). World Science and Technology/Modernization of Traditional Chinese Medicine and Materia Medica 2020;22:242-7.

13. Song Y, Yao C, Yao Y, et al. XueBiJing Injection Versus Placebo for Critically Ill Patients With Severe Community-Acquired Pneumonia: A Randomized Controlled Trial. Crit Care Med 2019;47:e735-e743.

14. China Association Of Integrative Medicine Emergency Medicine Committee, Editorial Committee Of Chinese Journal Of Integrated Traditional And Western Medicine, Li Z, Wang D, et al. Expert consensus on diagnosis and treatment of septic shock with integrated traditional Chinese and Western medicine. Zhonghua Wei Zhong Bing Ji Jiu Yi Xue 2019;31:1317-23.

15. Zhou L, Liu K, Liu HG. Cause analysis and treatment strategies of "recurrence" with novel coronavirus pneumonia (COVID-19) patients after discharge from hospital. Zhonghua Jie He He Hu Xi Za Zhi 2020;43:281-4.

16. Wan S, Xiang Y, Fang W, et al. Clinical features and treatment of COVID-19 patients in northeast Chongqing. J Med Virol 2020;92:797-806.

17. Organization WH. Clinical management of severe acute respiratory infection when novel coronavirus (2019$\mathrm{nCoV}$ ) infection is suspected: interim guidance. World Health Organization. 28 January 2020.

18. Chu CM, Cheng VC, Hung IF, et al. Role of lopinavir/ ritonavir in the treatment of SARS: initial virological and clinical findings. Thorax 2004;59:252-6.

19. Arabi YM, Alothman A, Balkhy HH, et al. Treatment of Middle East Respiratory Syndrome with a combination of lopinavir-ritonavir and interferon- $\beta 1 \mathrm{~b}$ (MIRACLE trial): study protocol for a randomized controlled trial. Trials 2018;19:81.

20. Cao B, Wang Y, Wen D, et al. A Trial of LopinavirRitonavir in Adults Hospitalized with Severe Covid-19. N Engl J Med 2020;382:1787-99.

21. Gao J, Tian Z, Yang X. Breakthrough: Chloroquine phosphate has shown apparent efficacy in treatment of
COVID-19 associated pneumonia in clinical studies. Biosci Trends 2020;14:72-3.

22. Zhang SW, Sun CD, Wen Y, et al. Effect of treatment with Xuebijing injection on serum inflammatory mediators and Th1/2 of spleen in rats with sepsis. Zhongguo Wei Zhong Bing Ji Jiu Yi Xue 2006;18:673-6.

23. Chen S, Dai G, Hu J, et al. Discovery of Xuebijing Injection Exhibiting Protective Efficacy on Sepsis by Inhibiting the Expression of HMGB1 in Septic Rat Model Designed by Cecal Ligation and Puncture. Am J Ther 2016;23:e1819-e1825.

24. Jiang M, Zhou M, Han Y, et al. Identification of NF$\kappa \mathrm{B}$ Inhibitors in Xuebijing injection for sepsis treatment based on bioactivity-integrated UPLC-Q/TOF. J Ethnopharmacol 2013;147:426-33.

25. He XD, Wang Y, Wu Q, et al. Xuebijing Protects Rats from Sepsis Challenged with Acinetobacter baumannii by Promoting Annexin A1 Expression and Inhibiting Proinflammatory Cytokines Secretion. Evid Based Complement Alternat Med 2013;2013:804940.

26. Wang Q, Wu X, Tong X, et al. Xuebijing Ameliorates Sepsis-Induced Lung Injury by Downregulating HMGB1 and RAGE Expressions in Mice. Evid Based Complement Alternat Med 2015;2015:860259.

27. Wan S, Yi Q, Fan S, et al. Relationships among lymphocyte subsets, cytokines, and the pulmonary inflammation index in coronavirus (COVID-19) infected patients. Br J Haematol 2020;189:428-37.

28. Zhou Y, Fu B, Zheng X, et al. Aberrant pathogenic GM$\mathrm{CSF}+\mathrm{T}$ cells and inflammatory CD14+CD16+monocytes in severe pulmonary syndrome patients of a new coronavirus. biorxiv 2020. doi: https://doi. org/10.1101/2020.02.12.945576.

29. Guan WJ, Ni ZY, Hu Y, et al. Clinical Characteristics of Coronavirus Disease 2019 in China. N Engl J Med 2020;382:1708-20.

30. Tanaka T, Narazaki M, Kishimoto T. Immunotherapeutic implications of IL-6 blockade for cytokine storm. Immunotherapy 2016;8:959-70.

31. Zheng R, Wang H, Liu Z, et al. A real-world study on adverse drug reactions to Xuebijing injection: hospital intensive monitoring based on 93 hospitals (31,913 cases). Ann Transl Med 2019;7:117.

32. Grupp SA, Kalos M, Barrett D, et al. Chimeric antigen receptor-modified $\mathrm{T}$ cells for acute lymphoid leukemia. N Engl J Med 2013;368:1509-18.

33. Monteleone G, Sarzi-Puttini PC, Ardizzone S. Preventing COVID-19-induced pneumonia with anticytokine 
therapy. Lancet Rheumatol 2020;2:e255-e256.

infections. Nat Med 2020;26:1200-4.

34. Long QX, Tang XJ, Shi QL, et al. Clinical and immunological assessment of asymptomatic SARS-CoV-2

(English Language Editor: J. Reynolds)

Cite this article as: Guo H, Zheng J, Huang G, Xiang Y, Lang C, Li B, Huang D, Sun Q, Luo Y, Zhang Y, Huang L, Fang W, Zheng Y, Wan S. Xuebijing injection in the treatment of COVID-19: a retrospective case-control study. Ann Palliat Med 2020;9(5):32353248. doi: $10.21037 / \mathrm{apm}-20-1478$ 